\title{
An Introduction To Evolutionary Game Theory
}

\author{
Tim Rees \\ UBC Department of Computer Science \\ trees@cs.ubc.ca
}

\begin{abstract}
Evolutionary game theory has grown into an active area of research that bridges concepts from biology, evolution, nonlinear dynamics, and game theory. The mechanisms necessary to conduct an evolutionary analysis of games are presented. Relations between evolutionary stable strategies and Nash equilibria are considered. Replicator dynamics are developed and applied to three relevant games. The analysis of example games is used to illustrate the weaknesses and strengths of the theory.
\end{abstract}

\section{INTRODUCTION}

Evolutionary game theory (EGT) has grown into a field that combines the principles of game theory, evolution, and dynamical systems to interpret the interactions of biological agents. Practitioners in the field have used the theory to explain biological phenomena successfully, but EGT can also be used to interpret classical games from a different perspective. This document introduces evolutionary game theory and presents an evolutionary approach to the analysis of games.

There are several basic components in the EGT analysis of games. Game agents and their strategies must be simulated with populations of players, the fitness of different strategies relative to the population must be computed, and a process to govern the evolution of the population must be defined. These simple components can be combined to yield highly complex solutions. Ideally, under the dynamical process the strategies of the populations of players will converge to some stable value. Evolutionary game theorists often claim the evolutionary solution of the game as the true definition of rational play.

The concept of simulating populations of players to determine rational play is not new. A similar idea was apparently suggested by Nash in his doctoral thesis. The real birth of EGT, though, is likely due to Maynard Smith [6]. Work by Cressman [1] focusses heavily on the stability analysis of games. In Weibul [7] a broader treatment of continuous and discrete replicator dynamics is given, but with few applications. Recent work by Hofbauer and Sigmund provide an excellent mathematical treatment of the topic with many examples [5].

This document is structured as follows. First, a background description of evolutionary game theory and its most important concepts and equations is given in section 2. The EGT analysis techniques are then applied to the analysis of some important games in section 3. A more general discussion of the results of EGT is given in section 4. Potential avenues for future explorations are described in section 5 , followed by concluding remarks in section 6 .

\section{BACKGROUND}

In this section the fundamental concepts behind EGT will be introduced. Evolutionary stable strategies, convergence criteria, and discrete and continuous replicator dynamics will be covered.Interested readers should refer to the detailed treatment given by Weibul [7].

\subsection{Evolutionary Game Theory}

Evolutionary game theory is a different approach to the classic analysis of games. Instead of directly calculating properties of a game, populations of players using different strategies are simulated and a process similar to natural selection is used to determine how the population evolves. Varying degrees of complexity are required to represent populations in multi-agent games with differing strategy spaces.

To be exact, consider a $n$-player game where the $i^{\text {th }}$ player has strategy space denoted by $S_{i}$. An EGT approach would be to model each agent by a population of players. The population for the $i^{t h}$ agent would then be partitioned into groups $E_{i 1}, E_{i 2}, \ldots, E_{i k}$ ( $k$ might be different for each population). Individuals in group $E_{i j}$ would all play the same (possibly mixed) strategy from $S_{i}$. The next step, then, would be to randomly play members of the populations against each other. The sub-populations that performed the best would grow, and those that did not perform well would shrink. The process of playing members of the populations randomly and refining the populations based on performance would be repeated indefinitely. Ideally the evolution would converge to some stable state for each population, which would represent a (possibly mixed) strategy best response for each agent. 
A special case is the symmetric two-player game. In a symmetric game payoff matrices and actions are identical for both agents. These games can be modelled by a single population of individuals playing against each other. When the game being played is asymmetric, a different population of players must be used to simulate each agent.

Throughout this document, the EGT approach will make use of the matrix-vector formulation of games. If $s_{1}, \ldots, s_{n} \in$ $S$ are the pure strategies available to a player, then that player's strategy will be denoted by the column vector $\vec{x}$. The $i^{t h}$ component of $\vec{x}$ gives the probability of playing strategy $s_{i}$. Playing a pure strategy $s_{j}$ is represented by the vector whose $j^{\text {th }}$ component is 1 , and all other components are 0 . When the payoff for a player is specified by a payoff matrix $A$, a player using strategy $\vec{x}$ against an opponent with strategy $\vec{y}$ will have payoff $\vec{x}^{T} A \vec{y}$.

There are several critical components to an EGT analysis. The natural selection process governing the evolution of populations requires a measure of fitness for different strategies, and the process itself must be carefully chosen. Before a full discussion of the evolution process, though, it is necessary to describe evolutionary stable strategies.

\subsection{Evolutionary Stable Strategies}

The analogue of a Nash equilibria in EGT is the evolutionary stable strategy (ESS). In fact, an ESS is a Nash equilibrium, but it is somewhat strengthened. ESS are a fundamental concept in evolutionary game theory and are treated carefully in any text on the subject.

A strategy $\vec{S}^{*}$ for the symmetric game with payoff matrix $A$ is an ESS if two conditions are met:

- $\vec{S}^{T} A \vec{S}^{*} \leq \vec{S}^{* T} A \vec{S}^{*}$, for all strategies $\vec{S} \neq \vec{S}^{*}$.

- $\vec{S}^{T} A \vec{S}<\vec{S}^{* T} A \vec{S}$ when equality in the first condition holds.

Clearly any strategy $\vec{S}^{*}$ satisfying these criteria is also a Nash equilibrium (since it is a best response to itself and the game is symmetric). In fact, an ESS is somewhat stronger than a weakly dominant strategy. Refinements to the ESS definition are required for asymmetric games and differing evolutionary models.

Loosely speaking, a population in an ESS is resistant to invasion by a small number of mutants playing a different strategy. This means that if a few individuals playing a different strategy were introduced into a population in an ESS state, the evolutionary selection process would eventually eliminate the invaders. As long as the invading population is sufficiently small (but non-zero), they would not be as fit as the original population, so they would become extinct through evolution. The selection process lies at the heart of EGT, and is presented next.

\subsection{Replicator Dynamics}

The natural selection process that determines how populations playing specific strategies evolve is known as the repli- cator dynamics. Slightly differing versions of these equations can be found in $[1,5,7]$. There are different replicator dynamics depending on the evolutionary model being used. This section will present discrete and continuous time versions of the equations for symmetric games, and a continuous equation for asymmetric games. Only two-player games will be considered.

The notation used in the replicator dynamics is continued from section 2.1. For each population representing an agent, a vector $\vec{x}$ is constructed with $i^{\text {th }}$ component equal to the frequency of the corresponding sub-population.

Before defining the replicator dynamics a notion of fitness is required. The fitness function specifies how successful each sub-population is, it is given by $f_{i}(\vec{x})$ and must be defined for each component of $\vec{x}$.

Symmetric normal form games are of particular interest. For these the definition of the fitness function is immediate. In the two player symmetric case (where the payoff matrix for each player and strategy space are identical), the fitness for $x_{i}$ is just $(A \vec{x})_{i}$. The subscript $i$ denotes the $i^{t h}$ component of the matrix-vector product. Note that this is just the expected utility of playing strategy $s_{i}$ against a player with a mixed strategy defined by the vector $\vec{x}$. The average fitness of the population is then $\vec{f}(\vec{x})=\vec{x}^{T} A \vec{x}$.

Once the fitness measure is defined the replicator dynamics can be described. When continuous mixing and interaction between generations in the model is acceptable, a continuous time dynamic can be used. In this case each component of the frequency vector is expressed as a continuous function of time. A differential equation that governs the growth of frequencies can then be defined. In keeping with Darwin's theory of evolution, the replicator dynamics specify that populations of the fittest individuals grow the fastest, while the least fit diminish the quickest. This gives rise to the continuous time replicator dynamics (taken from [5]) for a symmetric game:

$$
\dot{x}_{i}(t)=x_{i}(t)\left(f_{i}(\vec{x})-\bar{f}(\vec{x})\right) .
$$

Equation 1 rewards strategies that outperform the average by increasing their frequency, and penalizes poorly performing strategies by decreasing their frequency. It is important to note that if at any point in time the frequency of a particular strategy becomes 0 , it will remain there.

In many situations it is not appropriate to model the frequencies as continuous functions of time. Using a discrete model allows for the prevention of mixing between generations. The discrete dynamic must play the same role as the continuous version. Frequencies corresponding to fit strategies must increase, and those that correspond to unfit strategies must diminish. An example discrete dynamic taken from [7] is given below.

$$
x_{i}(t+1)=\frac{\alpha+f_{i}(\vec{x})}{\alpha+\bar{f}(\vec{x})} x_{i}(t)
$$


The parameter $\alpha$ performs the role of regulating the rate of growth or decay of strategies, large values will lead to slower convergence, smaller values give faster, but less stable convergence.

The two-player asymmetric normal form game requires special treatment. In this situation each player will have a distinct payoff matrix, and must be represented by a different population of simulated players. The fitness is not measured by play within the same population, but rather play between populations. Assuming that player 1 has $n$ pure strategies denoted by $s_{11}, \ldots, s_{1 n}$, and player 2 has $m$ pure strategies denoted by $s_{21}, \ldots, s_{2 m}$, then the payoff matrices can be written as $A \in \Re^{n \times m}$, and $B \in \Re^{m \times n}$. The $n \times 1$ strategy vector for the first player is represented by $\vec{x}$, and the $m \times 1$ strategy vector for the second player is represented by $\vec{y}$. The fitness of an individual playing strategy $s_{1 i}$ will be $f_{1 i}=(A \vec{y})_{i}$. The average fitness of the first population will be $\bar{f}_{1}=\vec{x}^{T} A \vec{y}$. Similarly the fitness of strategy $s_{2 i}$ will be $f_{2 i}=(B \vec{x})_{i}$, and the average fitness for the second population will be $\bar{f}_{2}=\vec{y}^{T} B \vec{x}$.

A continuous replicator dynamic can be defined for the twoplayer asymmetric normal form game, but a dynamic must be specified for each player. Using the same reasoning as for the development of equation 1, the following asymmetric continuous time dynamic can be used (as defined in [5]).

$$
\begin{aligned}
& \dot{x}_{i}=x_{i}\left((A \vec{y})_{1}-\vec{x}^{T} A \vec{y}\right) \\
& \dot{y}_{i}=y_{i}\left((B \vec{x})_{1}-\vec{y}^{T} B \vec{x}\right)
\end{aligned}
$$

It is important to note that the Nash equilibria of a game are the fixed points of each of the dynamics presented above. To see this for the symmetric case, consider that in a mixedstrategy Nash equilibrium $(A \vec{x})_{i}$ is constant, and since the sum of the components of $\vec{x}$ is $1,(A \vec{x})_{i}=\vec{x}^{T} A \vec{x}$. A similar argument holds for the asymmetric case. The fixed points of the system do not change in time. This means if a population ever reaches a state that is a Nash equilibrium, it will remain there. This does not guarantee that populations will converge to a Nash equilibrium.

The replicator dynamics, while intuitive, do not generally lead to systems that are easy to solve. The role of the selection process in EGT will become more clear in the next section through the presentation of examples.

\section{EVOLUTIONARY ANALYSIS OF GAMES}

This section will analyze three interesting games that illustrate some of the strengths and weaknesses of EGT. The first game, the prisoner's dilemma, should be familiar to all. The second games is analyzed to illustrate some of the convergence problems of the replicator dynamics. A final game is presented to demonstrate some of the power of EGT in explaining biological phenomena.

\subsection{The Prisoner's Dilemma}

The classic two-player prisoner's dilemma is an important game that warrants an investigation with EGT. The game is expressed in normal form below.

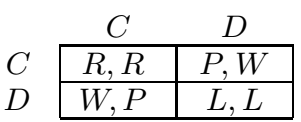

Here $C$ denotes cooperation between the players and $D$ denotes defection. The reward $R$ is attained when both players cooperate, the loser's reward $L$ is obtained when both players defect. When one player cooperates and the other defects, the cooperator receives $P$ (for punishment) and the defector receives $W$ (for winning).It is assumed that $P<L<R<W$. Note that the game is symmetric, both players have the same payoff matrix.

When the game is repeated a finite number of times, backwards induction can be used to show that defection at each stage by both players is a dominant strategy. How can EGT be used to interpret the game?

The EGT analysis of the game requires careful definitions of the payoff matrix and strategy vectors for players. The payoff matrix for each player is $A=\left(\begin{array}{cc}R & P \\ W & L\end{array}\right)$. Since there are only two pure strategies, a population with two groups can be constructed. Denote the frequency of cooperators by $x$, the frequency of defectors in the population is then $1-x$, so the strategy frequency vector is $\vec{x}=\left(\begin{array}{c}x \\ 1-x\end{array}\right)$. It is sufficient to study the evolution of the cooperator frequency, since the defector frequency falls immediately from it.

To simplify analysis consider the concrete example $A=$ $\left(\begin{array}{rr}-1 & -20 \\ 0 & -10\end{array}\right)$. This choice is analytically convenient but not exceptional, the same results will hold for any $P, L, R, W$ as long as the ordering does not change. The average fitness of the population is then $\vec{x}^{T} A \vec{x}=9 x^{2}-10$. The fitness of the cooperators is $(A \vec{x})_{1}=19 x-20$. Now, letting $\vec{x}=\vec{x}(t)$, the replicator dynamics for the cooperators (the first component of $\vec{x}$ ) becomes:

$$
\begin{aligned}
\dot{x} & =x\left((A \vec{x})_{1}-\vec{x}^{T} A \vec{x}\right) \\
& =x\left(19 x-20-9 x^{2}+10\right) \\
& =-x(9 x-10)(x-1) .
\end{aligned}
$$

A plot of the polynomial $\dot{x}$ is shown in Figure 3.1. Note that $\forall x \in(0,1), \dot{x}<0$. That is, the frequency of cooperators is strictly decreasing. Under the replicator dynamics the frequency of cooperators will converge to 0 , leaving a population purely composed of defectors. This indicates that a population of purely defecting players is a fixed point of the system, and hence $D$ is a Nash equilibrium. The convergence to a stable point is due to the fact that the pure strategy $D$ is an ESS. Under the given dynamic, the introduction of any number of cooperators to the population will result in the extinction of those cooperators and return to the stable state.

Extending the above analysis to the iterated prisoner's dilemma is complicated. The main problem is the exponential growth in the strategy space with the number of iterations. To reduce the complexity of the analysis an EGT approach can be applied to a small part of the strategy space. In [5], Hofbauer and Sigmund propose expressing strategies for each 


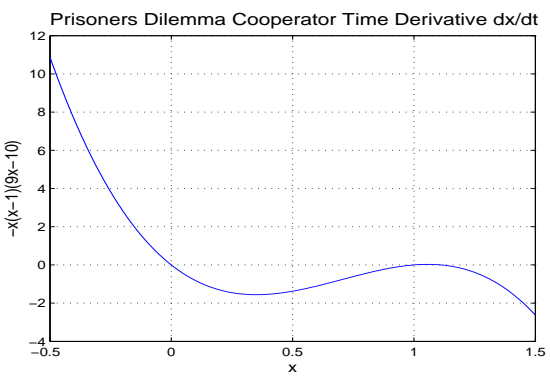

Figure 1: The time derivative $\dot{x}$ for the cooperators in the prisoner's dilemma, note that $\dot{x}$ is negative in the interval $(0,1)$, and since $x$ is a frequency this is the only region of interest.

player as a triple $(y, p, q)$, where $y$ denotes the probability of initially cooperating, $p$ denotes the probability of cooperating given an opponent's prior cooperation, and $q$ denotes the probability of cooperating given an opponent's prior defection. Such a triple, while limited, is sufficient to express the Tit for Tat strategy, playing either pure strategy at each stage game, and many others.

Analysis of the iterated prisoner's dilemma using this representation and the replicator dynamics leads to coupled nonlinear differential equations for each of $y, p, q$. The analysis, while tedious, is not very informative, populations do not converge to a fixed point and there is no known optimal strategy for the repeated game. The interested reader is referred to Hofbauer and Sigmund [5].

\subsection{The Battle of the Sexes}

The battle of the sexes is an interesting game that gives some insight into the mating habits of certain animals. A thorough treatment of the game can be found in [5]. Consider a two-player game with two strategies for each player. Males must choose between being faithful $(F)$, and unfaithful $(U)$. Female players must choose between playing hard-to-get $(H)$ and being easy $(E)$. Producing an offspring generates $R$ units of reward (to both parents) but requires $C$ units to raise. Faithful males will stay with their mates and share the cost of raising their offspring, whereas unfaithful males will abandon their mates and force the female to pay the full cost. To prevent being abandoned, hard-to-get females will require a dating process at a cost of $D$ to both individuals. While faithful males will endure this, cheaters will not. It is assumed that $0<D<R<C<2(R-D)$. The game is expressed in normal form below.

\begin{tabular}{c|c|c|}
\multicolumn{2}{c}{$F$} & $U$ \\
\cline { 2 - 3 }$H$ & $R-\frac{C}{2}-D, R-\frac{C}{2}-D$ & 0,0 \\
\cline { 2 - 3 }$E$ & $R-\frac{C}{2}, R-\frac{C}{2}$ & $R-C, R$ \\
\cline { 2 - 3 } & &
\end{tabular}

The two payoff matrices are then: $A=\left(\begin{array}{cc}R-\frac{C}{2}-D & 0 \\ R-\frac{C}{2} & R-C\end{array}\right)$ for the females and $B=\left(\begin{array}{r}R-\frac{C}{2}-D R-\frac{C}{2} \\ 0\end{array}\right)$ for the males. The game is asymmetric, male and female players are not interchangeable since their action sets and payoffs differ.
It is clear that for the payoffs given, no pure strategy Nash equilibrium exists. There is, of course, a mixed strategy equilibrium. Having females play $H$ with probability $p$ and $E$ with probability $1-p$, it is found that $p=\frac{C}{2(R-D)}$ will make the males indifferent to their actions. Similarly, when males play $F$ with probability $q=\frac{R-C}{R-C-D}$, and $U$ with probability $1-q$, females will be indifferent. The mixed-strategy Nash equilibrium is then defined by the two strategy vectors $\vec{p}_{N}=\left(\begin{array}{c}p_{N} \\ 1-p_{N}\end{array}\right)$, and $\vec{q}_{N}=\left(\begin{array}{c}q_{N} \\ 1-q_{N}\end{array}\right)$, with $p_{N}=\frac{C}{2(R-D)}$ and $q_{N}=\frac{R-C}{R-C-D}$.

Will a population of players reach this equilibrium and remain there? The evolutionary analysis of this game is nontrivial. Each player must be represented by a distinct population of players, pairs are then randomly drawn from the two populations and played against each other. A stability analysis of the differential equations generated by the (asymmetric) replicator equations is also required.

Since each player only has two strategies it is sufficient to analyze the frequency of playing $H$ for females and $F$ for males, from which the frequencies for $E$ and $U$ are immediate. Using $\vec{p}=\left(\begin{array}{c}p \\ 1-p\end{array}\right)$, and $\vec{q}=\left(\begin{array}{c}q \\ 1-q\end{array}\right)$ as the strategy vectors for females and males respectively, the asymmetric replicator equations are given below.

$$
\begin{aligned}
& \dot{p}=p\left((A \vec{q})_{1}-\vec{p}^{T} A \vec{q}\right) \\
& \dot{q}=q\left((B \vec{p})_{1}-\vec{q}^{T} B \vec{p}\right)
\end{aligned}
$$

To observe the behavior pictorially, consider the situation that $R=5, C=6$, and $D=1$. The Nash equilibrium is based on the mixing strategy $\vec{p}_{N}=\left(\begin{array}{l}0.75 \\ 0.25\end{array}\right)$ for females, and $\vec{q}_{N}=\left(\begin{array}{l}0.5 \\ 0.5\end{array}\right)$ for males. It is important to point out that the Nash equilibrium is a fixed point of equation 4. If $\vec{p}_{N}, \vec{q}_{N}$ are substituted into the equation it is found that $\dot{p}=\dot{q}=0$. This means if populations were ever in the Nash equilibrium state, it would not change over time under the replicator dynamics.

Figure 3.2 shows that while $p_{N}, q_{N}$ may be a fixed point of the system, it is not globally stable. In fact, initial $p$ and $q$ values arbitrarily close to the Nash equilibrium will still diverge from the fixed point under equation 4 . For this game, there is no ESS. As shown in [5], as long as the ordering on the parameters $R, C, D$ is unchanged, trajectories will at best be cyclic, but never converge.

This game illustrates some important points and potential problems with EGT. What does it mean when an ESS does not exist? If the ES is a supposed improvement on the Nash equilibrium, and no ESS exists, then what constitutes rational play? Does the EGT analysis give any additional insight into the game? Some of these issues will be considered in section 4 .

\subsection{The Gender Ratio Game}

An interesting and informative example of the relevance of EGT to biological applications can be seen through the gender ratio game. The analysis helps to answer the question of why animal species generally have the same number of 
Evolutionary Divergence from a Nash Equilibria

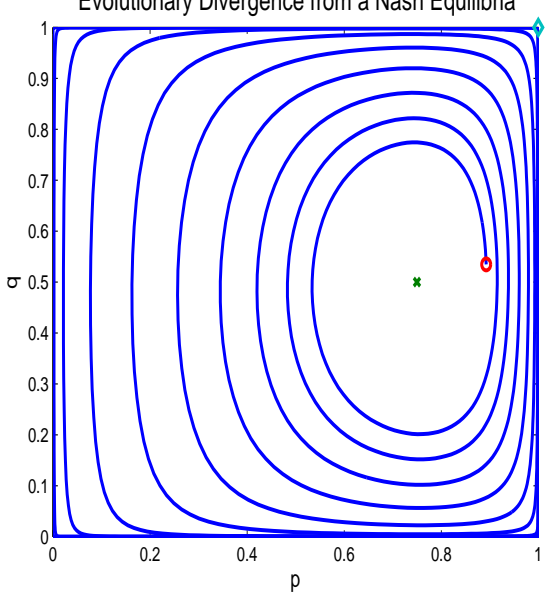

Figure 2: Populations diverge from the Nash equilibrium (denoted by ' $x$ '). A sample trajectory originating from ' $o$ ' spirals away from the equilibrium.

females and males. This problem is a classic result of EGT and the derivation presented here is based on the work of Cressman [1].

The game requires some basic assumptions, some of which will be addressed in section 4 . The assumptions are that the ability of the species to produce male or female children is determined purely by the females. The game is played by females and there are two strategies, to produce sons $S$ or daughters $D$. A strategy is represented by the vector $\vec{r}=$ $\left(\begin{array}{c}r \\ 1-r\end{array}\right)$, where $r \in[0,1]$ is the probability of giving birth to a son, and is known as the gender ratio. The gender ratio of an animal is purely determined by the ratio of its mother, so in this case strategies are inherited genetic properties instead of choices.

The model further assumes that all females will give birth to the same number of children, $C$. This is not unreasonable, the ability of an animal to produce children does not necessarily depend on the gender of the children it produces. The gender ratio does have a direct impact on the number of grandchildren a female can spawn, and this is used to measure the fitness of an individual.

To proceed with an EGT analysis, it is necessary to define a population of players. Initially, let there be $N$ females that can be separated into $k$ groups, where the $i^{\text {th }}$ group has a unique gender ratio $r_{i}$. If the number of individuals in a group is $N_{i}$ then denote the frequency by $x_{i}=N_{i} / N$. The average gender ratio of the system is given by $\bar{r}=\sum_{i=1}^{k} x_{i} r_{i}$. Before analyzing how the replicator equations will evolve the frequencies, more details on the fitness function are needed.

The initial population of $N$ females will each give birth to $C$ children, so that a total of $N C$ children will be born. The average gender ratio will dictate that there be $\bar{r} N C$ male children, and $(1-\bar{r}) N C$ female children. Now, consider a female with gender ratio $r$, her $(1-r) C$ daughters will each produce $C$ children for a contribution of $(1-r) C^{2}$ grandchildren. It is assumed her sons will mate randomly

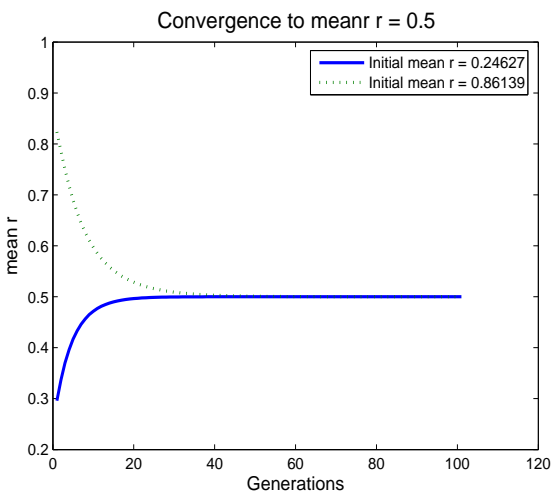

Figure 3: Regardless of the initial average gender ratio (as long as $\bar{r} \in(0,1)$ ) the population converges to the globally stable ratio $r=1 / 2$. Note that an initial gender ratio of $\bar{r}=0$ or $\bar{r}=1$ would lead to the extinction of the species within one generation.

with the population, produce the same number of children on expectation, and that the population is large enough that siblings do not mate. Then each son should produce a total of: $\frac{1}{\bar{r} N}(1-\bar{r}) N_{f} C$ children. Since the female in question will have $r C$ sons they will produce a total of $r \frac{1-\bar{r}}{\bar{r}} C^{2}$ children. Thus the total number of grandchildren (and thus fitness) of a female with gender ratio $r$ will be $f(r)=C^{2}\left(1-r+r \frac{1-\bar{r}}{\bar{r}}\right)$. The average fitness of the population is $\bar{f}=f(\bar{r})=2 C^{2}(1-$ $\bar{r})$.

The next consideration in the EGT analysis is the choice of a replicator dynamic. Since mixing between generations should not occur, a continuous dynamic is unsuitable. This leaves the discrete dynamics of section 2.3. Evolution of populations is given by:

$$
x_{i}(t+1)=\frac{\alpha+f_{i}(\vec{r})}{\alpha+\bar{f}(\vec{r})} x_{i}(t),
$$

where $\alpha$ is a small constant controlling the growth rate.

Clearly the $i^{t h}$ sub-population will grow whenever $f_{i}(\vec{r})>$ $f(\bar{r})$. Thus whenever $C^{2}\left(1-r_{i}+r_{i} \frac{1-\bar{r}}{\bar{r}}\right)>2 C^{2}(1-\bar{r})$, or $r_{i}(1-2 \bar{r})>\bar{r}(1-2 \bar{r}), x_{i}$ will increase. When $\bar{r}<1 / 2$ mixed strategies with $r_{i}>\bar{r}$ will flourish, and those with $r_{i}<\bar{r}$ will diminish. Similarly if $\bar{r}>1 / 2$, all populations with mixed strategies $r_{i}<\bar{r}$ will grow, and those populations with $r_{i}>$ $\bar{r}$ will shrink. When $\bar{r}=1 / 2$, the replicator equation will reduce to $x_{i}(t+1)=x_{i}(t)$ for each group $i$.

Figure 3.3 shows the convergence of the mean gender ratio $\bar{r}$ to $1 / 2$ under two different initial conditions. The ratio $\bar{r}=1 / 2$ is an ESS. As long as the mean gender ratio lies in the interval $(0,1)$ initially, it will converge to $1 / 2$. The rate of convergence is controlled by the $\alpha$ parameter. The introduction of a small number of animals with a different gender ratio will not change the balance, the replicator equation will balance the population to reinforce the ESS.

\section{DISCUSSION}


Having seen a few examples of EGT in action, it is important to discuss the strengths and weaknesses of the theory. The first major concern is that while Nash equilibria are fixed points of the replicator dynamics, they are not generally stable, as was seen in the battle of the sexes. Convergence to a Nash equilibrium requires asymptotic stability of the fixed point, but it is also dependent on the initial conditions of the populations. This means that given two populations with different initial distributions across the strategies, the replicator dynamics might lead to two different stable states. From a game theory perspective, this may not be desirable, but it could be useful biologically. The same species in slightly different conditions likely would evolve to different states.

The passage of genetic information (and thus strategies) used in the models of section 3 has raised concern with biologists. In each example a haploid model was used where future populations inherited their strategies purely from one parent. In $[1,5]$ mechanisms are described for a more realistic modelling of the passage of genetics from generation to generation, but they further complicate an already cumbersome model.

The examples in the previous section illustrated some computational problems about evolutionary game theory. Even the analysis of $2 \times 2$ games was seen to be non-trivial. Coupled nonlinear differential equations arise from the replicator dynamics for simple systems. The extension to games with more players and more pure strategies would greatly complicate analysis. Explicitly solving these equations would be impractical, and the asymptotic stability analysis of high dimensional system is still an open problem in mathematics [5]. The numerical stability of the replicator dynamics is also of concern. The numerical stability of the discrete dynamic is of special concern, and Cressman even cautions against its use [1].

With all of these concerns, what can EGT contribute to game theory? Perhaps EGT offers a more realistic interpretation of the way players with bounded rationality interact in a game. EGT mimics tournament play where losing strategies are eliminated and winning strategies remain. If no clear winning state can be found, does this suggest the game is too complicated for bounded individuals? The results do suggest, though, that even a single irrational player could destabilize a population playing in an equilibrium state.

Clearly the heart of EGT lies in the stability analysis of differential and difference equations and the implications for games. It is likely that the main contribution EGT can make to game theory will be in refining the definition of rationality. It is more likely that the main contributions of EGT will be in the analysis of simple biological systems to explain phenomena such as the gender ratio.

\section{FUTURE WORK}

Evolutionary game theory is intended to help interpret biological interactions, and it is in that area that future research might be most rewarding. The application of the tools developed in EGT could prove very helpful in explaining common phenomena (such as the gender ratio). The dynamical mod- els presented might not generally be suitable for analyzing species with long lifespan and gestation periods. The real power of EGT might lie in the analysis of games involving viruses and bacteria that can evolve quickly. A sufficient background in biology would be required for such a study.

An alternative exploration might be to focus on the stability nature of EGT for certain classes of games. Outside the realm of two player, two strategy games little is known about the stability and convergence of the replicator equations, and open problems exist [5]. Such an analysis would be challenging mathematically, but could contribute to the understanding of how bounded individuals play complex games.

\section{CONCLUSIONS}

Evolutionary game theory combines concepts from nonlinear dynamics, game theory and biology to provide an alternative analysis of games. The heart of EGT, though, lies in the stability analysis of the differential and difference equations that arise from the replicator dynamics. It was seen that the analysis of simple two player games was non-trivial and lead to nonlinear dynamics. The extension of EGT to larger, more complicated games would be a very difficult task. It was also seen, though, that EGT does have the potential to explain certain biological phenomena, such as the gender ratio. Evolutionary stable strategies were seen to be related to Nash equilibria, but there lack of existence or non-uniqueness in some games raises concerns.

\section{REFERENCES}

[1] Ross Cressman. The Stability Concept of Evolutionary Game Theory: A Dynamic Approach. Springer-Verlag, New York, 1992.

[2] Richard H. Day. Complex Economic Dynamics Volume 1. MIT Press, Cambridge MAy, 1994.

[3] Richard H. Day. Complex Economic Dynamics Volume 2. MIT Press, Cambridge MAy, 1999.

[4] Drew Fudenberg and Jean Tirole. Game Theory. MIT Press, Cambridge, MA, 1991.

[5] Joseph Hofbauer and Karl Sigmund. Evolutionary Games and Replicator Dynamics. Cambridge University Press, Cambridge, UK, 1998.

[6] Maynard Smith. Evolution And The Theory of Games. Cambridge University Press, Cambridge, UK, 1982.

[7] Jorgen Weibul. Evolutionary Game Theory. MIT Press, Cambridge, MA, 1995. 